\title{
Application of the method of robust estimation by posterior variance in detecting the raw error of geodetic control network
}

\author{
Khanh Quoc Pham*, Thanh Kim Thi Nguyen \\ Hanoi University of Mining and Geology, Hanoi, Vietnam
}

\begin{abstract}
ARTICLE INFO
ABSTRACT

Article history:

Received $19^{\text {th }}$ Nov. 2020

Revised 17th Mar. 2021

Accepted 09th Apr. 2021

Keywords:

Posterior variance.

Raw error,

Robust estimation,

Structural monitoring.

If the raw error appears in set of measuring data, it affects significantly on adjustment results and displacement values of monitoring points, thus conclusion about displacement of works is incorrect. The method of robust estimation by posterior variance for detecting the raw error bases on principle of choosing weight of robust estimation, this is the other type that belong to the least square statistical estimation, which is used to process the measuring data with raw error when they were given into random model of the adjustment problem. Through processing data of Son La hydroelectricity construction network, the obtained result proved that the method good efficiency, it not only finds the measuring value that contains the raw error, but also determines the value of the raw error nearly accurately, moreover, it is able to detect many raw error in the set of data.
\end{abstract}

Copyright (C) 2021 Hanoi University of Mining and Geology. All rights reserved.

${ }^{*}$ Corresponding author

E - mail: phamquockhanh@humg.edu.vn

DOI: 10.46326/JMES.2021.62(2).06 


\title{
Tạp chí Khoa học Kỹ thuật Mỏ - Địa chất
}

Trang điện tử: http://tapchi.humg.edu.vn

\section{Ứng dụng phương pháp ước lượng vững theo phương sai hậu nghiệm phát hiện sai số thô trong lưới khống chế trắc địa}

\author{
Phạm Quoc Khanh *, Nguyễn Thị Kim Thanh
}

Trường đại học Mỏ - Địa chất, Hà Nội, Việt Nam

\begin{abstract}
THÔNG TIN BÀI BÁO
Quá trình:

Nhận bài 19/11/2020

Sửa xong $17 / 3 / 2021$

Chap nhận đăng 09/4/2021

Tù khóa:

Lưới thi công,

Phương sai hậu nghiệm,

Sai số thô,

Ước lượng vững.

\section{TÓM TẮT}

Sai số thô nếu tồn tại trong tập sốliệu đo ngoại nghiẹp thì sẽ ảnh hưởng lớn đến kết quả sau bình sai và lượng chuyển dịch của các điểm quan trắc, tù̀ đó dẫn đến nhận định và kết luận không đúng về chuyển dịch của công trình. Phương pháp ước lượng theo phương sai hậu nghiệm phát hiện sai số thô dựa trên nguyên lý thay thế chọn trọng số ước lượng vưng, đây là một phương pháp khác ước lượng thống kê số bình phương nhỏ nhất nhằm vào tri đo có chứa sai số thô khi tri đo này được đưa vào mô hình ngẫu nhiên của bài toán bình sai. Thông qua xử lý số liệu lưới thi công thủy điện Sơn La, kết quả tính toán chứng tỏ phương pháp này có hiệu quả tốt, ngoài khả năng xác định được trị đo có chứa sai số thô, còn có thể xác định gần chính xác giá trị của sai số thồ có trong trị đo, hơn nữa xác định được không chỉ một mà nhiều sai số thô trong tập trị đo.
\end{abstract}

C 2021 Trường Đại học Mỏ - Địa chất. Tất cả các quyền được bảo đảm.

\section{Mở đầu}

Trị đo có chứa sai số thô là trị đo có số hiệu chỉnh lớn hơn 3 lần sai số trung phương của máy đo, dãy trị đo một mạng lưới trắc địa gọi là tin cậy khi không có quá $10 \%$ trị đo có chứa sai số thô (Li Deren, Yuan Xiuli, 2012). Để tìm sai số thô trong tập trị đo ngoại nghiệp của một mạng lưới trắc địa, người làm trắc địa thường sử dụng phương trình điều kiện trong lưới để phát hiện sự bất thường trong nội bộ phương trình đó. Đối với mạng lưới lớn, nhiều trị đo và phương trình điều kiện thì phương pháp này không hiệu quả vì mất nhiều

\footnotetext{
*Tác giả liên hệ

E - mail: phamquockhanh@humg.edu.vn DOI: 10.46326/JMES.2021.62(2).06
}

thời gian và chỉ có thể tìm ra trị đo có chứa sai số thô khi các phương trình điều kiện có sự ràng buộc chặt chẽ với nhau. Phương pháp ước lượng vững (Robust estimation) do G.R.P. Box đề xuất từ những năm 1953 của thế kỷ trước và có ứng dụng rất rộng trong nhiều lĩnh vực như lý thuyết tối ưu (Baselga S., 2007), bình sai lưới GPS,... (Yang Yuanxi và nnk., 2005). Ước lượng vững chia làm ba loại cơ bản là ước lượng tự nhiên lớn nhất $(M)$, ước lượng tuyến tính sắp xếp thứ tự $(L)$ và ước lượng hạng $(R)$. Trong đó, ước lượng $M$ do Huber đề xuất năm 1964 và được Krarup và Kubit vận dụng được ứng dụng chủ yếu trong trắc địa vì có tác dụng chống nhiễu và loại trừ sai số thô (Wang Xinzhou và nnk., 2006). Cho đến nay, cùng với sự phát triển của thiết bị đo đạc và công nghệ, phương pháp ước lượng này vẫn được ứng dụng rộng rãi trên thế giới do tính hiệu quả của nó mang 
lại (Li Deren, Yuan Xiuli, 2012; Yetkin, 2018; Wang, Zhu, 2016; Yetkin, Berber, 2014; Guo J. và nnk., 2010). Tuy nhiên, nước ta có rất ít nghiên cứu liên quan đến vấn đề này, gần đây có nghiên cứu (Quoc Khanh Pham 2016; Anh Tuan Luu, 2016) nhưng vận dụng các hàm trọng số đã được nghiên cứu từ lâu.

Ước lượng vững đưa sai số thô vào ngay trong mô hình ngẫu nhiên của quy trình bình sai, dùng phương pháp lựa chọn hàm trọng số và cách tính lặp để từng bước thay thế trọng số trong quá trình bình sai, làm cho sai số thô có giá trị rất nhỏ, từ đó loại bỏ sai số thô. Có rất nhiều phương pháp ước lượng vững nhưng phương pháp thay thế chọn trọng số được ứng dụng phổ biến do có thuật toán dễ hiểu, đơn giản trong tính toán và lập trình. Hiện có một số hàm trọng số của các nhà khoa học trên thế giới đề xuất để tính toán trong phương pháp chọn trọng số như hàm Huber, hàm Denmark, hàm IGG,... (Wang Xinzhou và nnk., 2006). Trong bài báo này chỉ nghiên cứu và ứng dụng phương pháp ước lượng vững bằng phương sai hậu nghiệm trong phát hiện sai số thô quan trắc chuyển dịch công trình.

\section{Phương pháp thay thế chọn trọng số ước lượng vững}

\subsection{Phương pháp ước lượng vũ̃ng M thay thế chọn trọng số}

Giả thiết trị đo độc lập là $L_{n, 1}$, vector tham số chưa biết là $\widehat{X}_{t, 1}$, phương trình sai số và ma trận trọng số là (Wang Xinzhou và nnk., 2006).

$$
\begin{gathered}
V=A \hat{X}-L=\left[\begin{array}{c}
a_{1} \\
a_{2} \\
\vdots \\
a_{n}
\end{array}\right] \hat{X}-\left[\begin{array}{c}
l_{1} \\
l_{2} \\
\vdots \\
l_{n}
\end{array}\right] \\
P=\left[\begin{array}{llll}
p_{1} & & & \\
& p_{n} & & \\
& & \ddots & \\
& & & p_{n}
\end{array}\right]
\end{gathered}
$$

Trong đó: $a_{i}$ - vector hệ số của phương trình số hiệu chỉnh thứ $i$ kích thước $1 x t$ ( $t$ là số tham số chưa biết).

Từ phương trình sai số, hàm số $\rho\left(l_{i}, \hat{X}\right)$ của ước lượng $\mathrm{M}$ được biểu thị là:

$$
\rho\left(l_{i}, \hat{X}\right)=\rho\left(v_{i}\right)
$$

Trong trường hợp các trị đo không cùng độ chính xác, ước lượng M có dạng:

$$
\sum_{i=1}^{n} p_{i} \rho\left(v_{i}\right)=\sum_{i=1}^{n} p_{i} \rho\left(a_{i} \hat{X}-l_{i}\right)=\min
$$

Lấy đạo hàm biểu thức trên và ký hiệu $\varphi\left(v_{i}\right)=\frac{\partial \dot{\rho}}{\partial v_{i}}$, được:

$$
\sum_{i=1}^{n} p_{i} \rho\left(v_{i}\right) a_{i}=0
$$

Đặt:

$$
\bar{p}_{i}=p_{i} w_{i}, w_{i}=\frac{\varphi\left(v_{i}\right)}{v_{i}}
$$

Được:

$$
\sum_{i=1}^{n} a_{i}^{T} \bar{p}_{i} v_{i}=0
$$

hoặc:

$$
A^{T} \bar{P} V=0
$$

Phương trình chuẩn của ước lượng $\mathrm{M}$ là:

$$
A^{T} \bar{P} A \hat{X}-A^{T} \bar{P} L=0
$$

Trong đó: $\bar{P}$ - ma trận trọng số tương đương; $\bar{p}_{i}$ - phần tử trọng số tương đương. Do $\bar{P}$ là hàm của $V$, thông qua việc gán cho nó giá trị ban đầu, dùng phương pháp thay thế để ước lượng tham số $\widehat{X}$, ước lượng vững $M$ cuối cùng của tham số là:

$$
\hat{X}=\left(A^{T} \bar{P} A\right)^{-1} A^{T} \bar{P} L
$$

\subsection{Quy trình tính toán thay thế chọn trọng số}

Quy trình tính toán thực hiện theo các bước: a/ Lập phương trình sai số, lấy giá trị ban đầu của hệ số trọng số đều bằng 1 , tức đặt $w_{1}=w_{2}=$ $\cdots=w_{n}, W=E, E$ là ma trận đơn vị, thì $\bar{P}^{(0)}=P$, $P$ là ma trận trọng số của trị đo.

b/ Giải hệ phương trình chuẩn (7), được trị ước lượng lần thứ nhất của tham số $\hat{X}$ và số hiệu chỉnh $V$, tức

$$
\begin{cases}\hat{X}^{(1)}= & \left(A^{T} \bar{P} A\right)^{-1} A^{T} \bar{P} L \\ V^{(1)}= & A \hat{X}^{(1)}-L\end{cases}
$$

$\mathrm{c} / \mathrm{Tù} V^{(1)}$, xác định hệ số trọng số của các trị đo mới theo $\frac{\varphi\left(v_{i}\right)}{v_{i}}=w_{i}$, tạo nên ma trận trọng số 
tương đương mới $\bar{P}^{(1)}$ theo $\bar{p}_{i}=p_{i} w_{i}$, lại giải hệ phương trình (10), được trị ước lượng lần hai của tham số $\hat{X}$ và sai số $V$ là:

$$
\begin{cases}\hat{X}^{(2)}= & \left(A^{T} \bar{P}^{(1)} A\right)^{-1} A^{T} \bar{P}^{(1)} L \\ V^{(2)}= & A \hat{X}^{(2)}-L\end{cases}
$$

Trong đó: hệ số trọng số $\frac{\varphi\left(v_{i}\right)}{v_{i}}=w_{i}$ được xác định tùy thuộc vào hàm $\varphi\left(v_{i}\right)$ như hàm Huber, Denmark,... Trong bài báo này, hệ số trọng số $\omega_{i}$ được lấy theo phương sai hậu nghiệm do Li Deren đề xuất được trình bày ở mục 3 bên dưới.

$\mathrm{d} /$ Từ $V^{(2)}$ cấu tạo trọng số tương đương mới $\bar{P}^{(2)}$, lại giải hệ phương trình chuẩn, tính thay thế tương tự, cho đến khi giá trị sai lệch nghiệm của hai lần giải phù hợp với hạn sai theo yêu cầu thì dừng.

e/ Kết quả cuối cùng thu được:

$$
\left\{\begin{array}{lc}
\hat{X}^{(k)}= & \left(A^{T} \bar{P}^{(k-1)} A\right)^{-1} A^{T} \bar{P}^{(k-1)} L \\
V^{(k)}= & A \hat{X}^{(k)}-L
\end{array}\right.
$$

Khi chọn hàm số $\rho$ khác nhau sẽ tạo ra nhiều dạng khác nhau của hàm trọng số, độ lớn của $v_{i}$ và $w_{i}$ tỷ lệ nghịch với nhau; $\mathrm{v}_{\mathrm{i}}$ càng lớn, $\mathrm{w}_{\mathrm{i}}$ và $\bar{p}_{i}$ càng nhỏ. Qua thay thế nhiều lần, làm cho trọng số của trị đo có chứa sai số thô bằng 0 hoặc tiến tới 0 , làm cho trong bình sai nó không còn tác dụng. Mặt khác, sai số của trị đo tương ứng phản ánh giá trị sai số thô của trị đo đó nhưng hàm trọng số luôn là đại lượng thay đổi theo số hiệu chỉnh trong quá trình bình sai. Phương pháp thông qua thay đổi trọng số trị đo trong quá trình bình sai để thực hiện tính vững của tham số chính là phương pháp thay thế trọng số.

\section{3. Đánh giá độ chính xác thay thế chọn trọng số trong ước lượng vũ̃ng}

Phương pháp thay thế chọn trọng số sử dụng hàm số cải chính $V$ tạo thành hàm trọng số, dùng phương pháp thay thế để ước lượng tham số, cho nên khi đánh giá độ chính xác cần xem xét hai vấn đề: một là hàm trọng số không phải là hằng số, nó cũng là hàm của trị đo, do đó cần xem xét đến việc tính toán thay thế; thứ hai là việc dẫn ra được công thức đánh giá chính xác có tính chặt chẽ là rất phức tạp, không thuận tiện trong thực tế.

Công thức ước tính tham số của thay thế chọn trọng số và nguyên tắc bình sai không giống với ước lượng bình phương nhỏ nhất kinh điển, chỉ là dùng trọng số tương đương $\bar{P}$ thay thế trọng số trị đo nếu trọng số tương đương lựa chọn cuối cùng giả định là hằng số. Nếu không xem xét tính toán thay thế thì nó giống với ước lượng bình phương nhỏ nhất. Vì thế, cần có một hệ thống các công thức gần đúng để đánh giá độ chính xác sau tính toán.

Công thức gần đúng ước lượng phương sai trọng số đơn vị là:

$$
\hat{\sigma}_{0}^{2}=\frac{V^{T} \bar{P} V}{n-t}
$$

Công thức gần đúng ma trận hiệp trọng số đảo của tham số $\hat{X}$ là:

$$
Q_{\hat{X} \hat{X}}=\left(A^{T} \bar{P} A\right)^{-1} A^{T} \bar{P} Q \bar{P} A\left(A^{T} \bar{P} A\right)^{-1}
$$

Công thức gần đúng ma trận hiệp phương sai của tham số $\hat{X}$ :

$$
D_{\widehat{X X}}=\hat{\sigma}_{0}^{2} Q_{\hat{X} \hat{X}}
$$

Ma trận hiệp trọng số đảo của tham số $\hat{X}$ trong ước lượng bình phương nhỏ nhất là:

$$
Q_{\hat{X} \hat{X}}=\left(A^{T} \bar{P} A\right)^{-1}
$$

So sánh (13) với (15) thấy rằng, ma trận hiệp trọng số đảo của ước lượng vưng thay thế chọn trọng số và ước lượng bình phương nhỏ nhất không chỉ đơn giản là dùng $\bar{P}$ thay thế $\mathrm{P}$.

Đối với bình sai lưới tự do có số khuyết, công thức gần đúng tính phương sai trọng số đơn vị là:

$$
\hat{\sigma}_{0}^{2}=\frac{V^{T} \bar{P} V}{n-t}=\frac{V^{T} \bar{P} V}{n-R(A)}
$$

Gọi $Q_{C}$ là ma trận nghịch đảo của ma trận hệ số của $\hat{X}$ thì ma trận hiệp trọng số đảo của $\hat{X}$ là:

$$
Q_{\hat{X} \hat{X}}=Q_{C} A^{T} \bar{P} Q A Q_{C}
$$

\section{Phương pháp ước lượng vững theo phương sai hậu nghiệm}

Phương pháp này do Li Deren thuộc Đại học Vũ Hán (Trung Quốc) đề xuất (Li Deren Yuan Xiuli, 2012), coi sai số thô là một mẫu con của mẫu chuẩn với phương sai rất lớn và kỳ vọng bằng 0 , thông qua ước lượng phương sai hậu nghiệm của phương pháp bình phương nhỏ nhất, tìm được phương sai hậu nghiệm của trị đo. Sử dụng kiểm nghiệm phương sai để tìm ra trị đo có chênh lệch phương sai bất thường, sau đó theo định nghĩa trọng số kinh điển tỷ lệ nghịch với phương sai trị đo, cung cấp cho nó một trọng số nhỏ tương ứng 
để tiến hành bước tính toán tiếp theo, từng bước định vị sai số thô.

Giả thiết có nhiều nhóm trị đo, trong mỗi nhóm có cùng độ chính xác quan trắc, hàm trọng số của trị đo thứ j, nhóm thứ i là:

$$
w_{i j}=\left\{\begin{array}{l}
p_{i}=\frac{\hat{\sigma}_{0}^{2}}{\hat{\sigma}_{i}^{2}}, T_{i j}<F_{\alpha, 1, r_{i}} \\
\frac{\hat{\sigma}_{0}^{2} r_{i j}}{v_{i j}^{2}}, T_{i j} \geq F_{\alpha, 1, r_{i}}
\end{array}\right.
$$

Trong đó:

$$
T_{i j}=\frac{\hat{\sigma}_{i j}^{2}}{\hat{\sigma}_{i}^{2}}
$$

là lượng thống kê kiểm nghiệm của phân bố $F ; r_{i j}$ - mức đo thừa của trị đo thứ $\mathrm{j}$ nhóm $\mathrm{i}$.

Các phương pháp thay thế chọn trọng số ở trên mặc dù hình thức hàm số trọng số không giống nhau, nhưng khi chọn dùng phương pháp bình phương nhỏ nhất, đều yêu cầu khi $|v| \leq C$ thì hàm trọng số là 1 ; khi $|v|>C$, dùng một hàm trọng số biểu đạt $v$ làm cho nó nhỏ hơn 1 , mức độ của trọng số phụ thuộc vào hình thức cụ thể của hàm trọng số đã cho, khi $|v|$ đạt đến hoặc vượt quá giá trị giới hạn làm cho hàm trọng số bằng không, nghĩa là trị đo bị loại bỏ trong tính toán.

\section{Tính toán thực nghiệm}

\subsection{Giói thiệu lưới thực nghiệm}

Lưới mặt bằng thi công Thủy điện Sơn La, tại huyện Mường La được thiết kế dưới dạng tam giác đo góc- cạnh. Lưới thi công công trình như trong Hình 1 gồm 8 điểm mới lập ký hiệu là TC03, TC04, TC05, TC06, TC07, TC08, TC09, TC10 và được đo nối với 3 điểm đường chuyền hạng IV là PV04, PV08, PV11 thành lập trong giai đoạn khảo sát công trình. Lưới thi công được đo theo phương pháp tam giác góc cạnh với tổng số trị đo là 46, trong đó có 27 trị đo góc, 19 trị đo cạnh bằng máy toàn đạc điện tử có sai số trung phương đo góc là 1,5 ", sai số trung phương đo cạnh là $3 \pm 2 p p m$.

\subsection{Phương pháp thực hiện}

Để khảo sát xem phương pháp ước lượng vững có thể phát hiện được sai số thô trong lưới thi công thủy điện Sơn La như lý thuyết đã nghiên cứu hay không, dựa vào số liệu đo thực tế, nhận

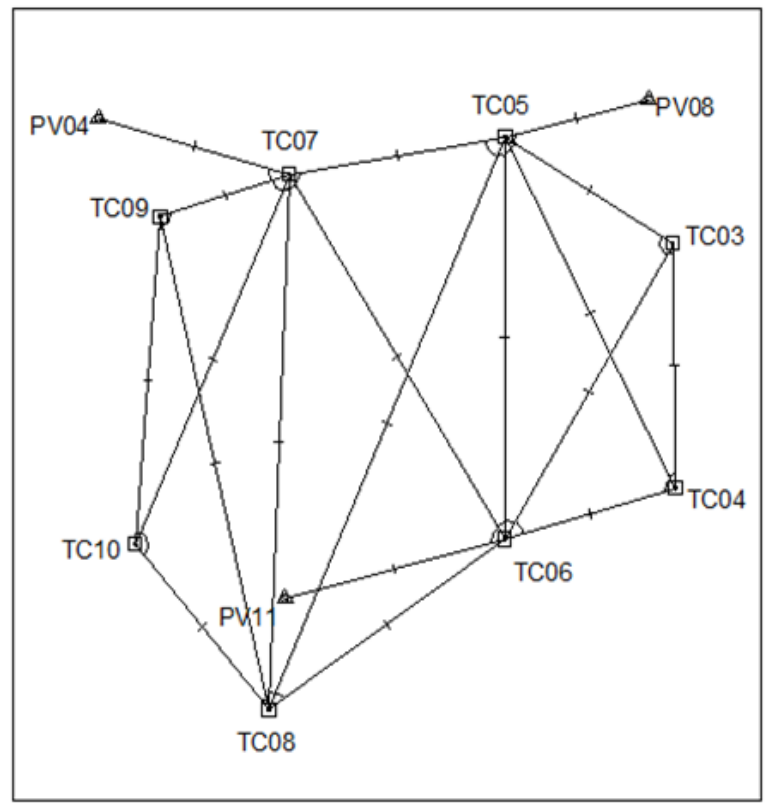

Hình 1. Sơ đồ lưới thi công thủy điện Sơn La.

thấy dãy trị đo này không có sai số thô trong trị đo. Chia thành hai trường hợp để thực nghiệm, thứ nhất là giả thiết trong lưới chỉ có một trị đo có chứa sai số thô, thứ hai là trong lưới có 3 trị đo có chứa sai số thô. Tổng trị đo trong lưới là 46 nên thực nghiệm giả thiết có không quá 4 trị đo có sai số thô để đảm bảo độ tin cậy.

\subsubsection{Trường hợp trong lưới chỉ có 1 trị đo có chứa sai số thô}

Dãy trị đo ban đầu của lưới thi công thủy điện Sơn La không có trị đo có chứa sai số thô, giả thiết có sai số thô tại trị đo cạnh thứ 2 (trị đo số 29) có sai số thô là $100 \mathrm{~mm}$ do nhập nhầm số liệu (số liệu đúng là 568,788 m bị nhập nhầm thành 568,888 $\mathrm{m})$. Đầu tiên bình sai lưới khi không có sai số thô, tiếp đó bình sai lưới có sai số thô; cuối cùng bình sai lưới kết hợp xác định sai số thô bằng ước lượng vững theo phương sai hậu nghiệm. Một phần kết quả được ghi trong Bảng 1.

4.2.2. Trường hợp trong lưới có 3 trị đo có chứa sai số thô

Giả thiết dãy trị đo trong lưới có 3 trị đo, gồm cả trị đo góc và trị đo cạnh, là trị đo góc số 2 (thêm 20 "), trị đo góc số 20 (giảm 20") và trị đo cạnh số 38 (tăng $60 \mathrm{~mm}$ ). Cũng tiến hành bình sai theo các bước như trường hợp 1. Một phần kết quả được ghi trong Bảng 2. Các bước thực hiện được thực hiện như sơ đồ Hình 2: 
Bảng 1. Trị đo, số hiệu chỉnh và trọng số tương úng thực nghiệm 1.

\begin{tabular}{|c|c|c|c|c|c|c|c|}
\hline TT & $\begin{array}{c}\text { Trị đo } \\
\text { Góc }(0 \text { “") } \\
\text { Cạnh }(\mathrm{m})\end{array}$ & $\begin{array}{c}\text { SHC SBS trị } \\
\text { đo không có } \\
\text { sai số thô }\end{array}$ & $\begin{array}{c}\text { SHC SBS } \\
\text { khi có sai } \\
\text { số thô }\end{array}$ & $\begin{array}{c}\text { SHC SBS sau } \\
\text { thay thế } \\
\text { trọng số }\end{array}$ & $\begin{array}{c}\text { Trọng số } \\
\text { ban đầu của } \\
\text { trị đo }\end{array}$ & Trọng số thay thế & Ghi chú \\
\hline$(1)$ & $(2)$ & $(3)$ & $(4)$ & $(5)$ & $(6)$ & $(7)$ & $(8)$ \\
\hline 1 & 315400.2 & 0,98 & $-0,01$ & 0,00 & 1,00 & 36985,7393 & \\
\hline$\ldots$ & $\ldots$ & $\ldots$ & $\ldots$ & $\ldots$ & $\ldots$ & $\ldots$ & \\
\hline 5 & 443053.7 & 0,00 & 0,00 & 0,00 & 1,00 & 18669214464139,0000 & \\
\hline 6 & 315325.6 & 0,57 & $-2,87$ & $-0,54$ & 1,00 & 0,6447 & \\
\hline$\ldots$ & $\ldots$ & $\ldots$ & $\ldots$ & $\ldots$ & $\ldots$ & $\ldots$ & \\
\hline 18 & 490253.5 & 0,04 & $-0,06$ & 0,02 & 1,00 & 838,1645 & \\
\hline 19 & 322355.2 & 0,07 & 0,00 & 0,00 & 1,00 & 14531543661769,0000 & \\
\hline 20 & 273430.9 & 0,00 & $-0,03$ & 0,03 & 1,00 & 1092,5633 & \\
\hline 21 & 153156.5 & $-0,76$ & $-0,79$ & $-0,79$ & 1,00 & 1,5951 & \\
\hline$\ldots$ & $\ldots$ & $\ldots$ & $\ldots$ & $\ldots$ & $\ldots$ & $\ldots$ & \\
\hline 29 & 568,788 & 2,97 & $-46,15$ & $-76,25$ & 0,13 & 0,0000 & Thêm $100 \mathrm{~mm}$ \\
\hline$\ldots$ & $\ldots$ & $\ldots$ & $\ldots$ & $\ldots$ & $\ldots$ & $\ldots$ & \\
\hline 43 & 579,202 & 0,00 & 0,00 & 0,00 & 0,13 & 38531439,2048 & \\
\hline 44 & 1387,020 & -2.88 & $-1,67$ & $-1,60$ & 0,07 & 0,0404 & \\
\hline 46 & 900,497 & 2.65 & 3,15 & 3,09 & 0,10 & 0,0310 & \\
\hline
\end{tabular}

Bảng 2. Trị đo, số hiệu chỉnh và trọng số tương ứng thực nghiệm 2.

\begin{tabular}{|c|c|c|c|c|c|c|c|}
\hline TT & $\begin{array}{c}\text { Trị đo } \\
\text { Góc (o") } \\
\text { Cạnh (m) }\end{array}$ & $\begin{array}{c}\text { SHC SBS } \\
\text { trị đo } \\
\text { không có } \\
\text { sai số thô }\end{array}$ & $\begin{array}{c}\text { SHC SBS } \\
\text { khi có sai } \\
\text { số thô }\end{array}$ & $\begin{array}{c}\text { SHC SBS sau } \\
\text { thay thế trọng } \\
\text { số }\end{array}$ & $\begin{array}{c}\text { Trọng số } \\
\text { ban đầu của } \\
\text { trị đo }\end{array}$ & Trọng số thay thế & Ghi chú \\
\hline$(1)$ & $(2)$ & $(3)$ & $(4)$ & $(5)$ & $(6)$ & $(7)$ & $(8)$ \\
\hline 1 & 315400.2 & 0,98 & 1,43 & 0,91 & 1,00 & 0,76206 & \\
\hline 2 & 893610.7 & 0,29 & $-11,40$ & $-14,72$ & 1,00 & 0,00596 & Thêm 20" \\
\hline 3 & 782011.6 & 0,78 & 2,69 & 2,12 & 1,00 & 0,17575 & \\
\hline$\ldots$ & $\ldots$ & $\ldots$ & $\ldots$ & $\ldots$ & $\ldots$ & $\ldots$ & \\
\hline 14 & 430925.5 & $-0,72$ & 1,08 & 0,15 & 1,00 & 6,05589 & \\
\hline 15 & 670527.8 & $-0,96$ & $-1,02$ & $-0,41$ & 1,00 & 2,41750 & \\
\hline 16 & 342746.8 & 0,86 & 5,46 & 2,24 & 1,00 & 0,08183 & \\
\hline 17 & 213846.5 & 0,16 & $-0,85$ & 0,01 & 1,00 & 154,50516 & \\
\hline 18 & 490253.5 & 0,04 & 0,17 & $-0,02$ & 1,00 & 259,04049 & \\
\hline 19 & 322355.2 & 0,07 & 0,00 & 0,00 & 1,00 & 50127574335569,0000 & \\
\hline 20 & 273430.9 & 0,00 & 13,67 & 16,27 & 1,00 & 0,00450 & Giảm $20 "$ \\
\hline 21 & 153156.5 & $-0,76$ & $-0,22$ & $-0,25$ & 1,00 & 18,20831 & \\
\hline 22 & 212534.8 & $-0,35$ & $-1,86$ & $-1,21$ & 1,00 & 0,44460 & \\
\hline 23 & 320752.8 & 0,35 & 0,98 & 0,36 & 1,00 & 2,85138 & \\
\hline 24 & 934623.7 & 0,36 & 0,69 & 0,06 & 1,00 & 23,15116 & \\
\hline$\ldots$ & $\ldots$ & $\ldots$ & $\ldots$ & $\ldots$ & $\ldots$ & & $\ldots$ \\
\hline 36 & 430,687 & 0,00 & 0,00 & 0,00 & 0,15 & 62256247040950,0000 & \\
\hline 37 & 1180,421 & $-0,89$ & $-1,02$ & $-2,40$ & 0,08 & 0,03191 & \\
\hline 38 & 830,339 & $-6,63$ & $-27,36$ & $-47,26$ & 0,10 & 0,00008 & Thêm $60 \mathrm{~mm}$ \\
\hline 39 & 660,814 & 0,00 & 0,00 & 0,00 & 0,12 & 38759314,08575 & \\
\hline$\ldots$ & $\ldots$ & $\ldots$ & $\ldots$ & $\ldots$ & $\ldots$ & & $\ldots$ \\
\hline 45 & 598,068 & 3,69 & 10,81 & 11,31 & 0,13 & 0,01182 & \\
\hline 46 & 900,497 & 2,65 & $-6,13$ & $-2,25$ & 0,10 & 0,01592 & \\
\hline & & & & & & & \\
\hline
\end{tabular}




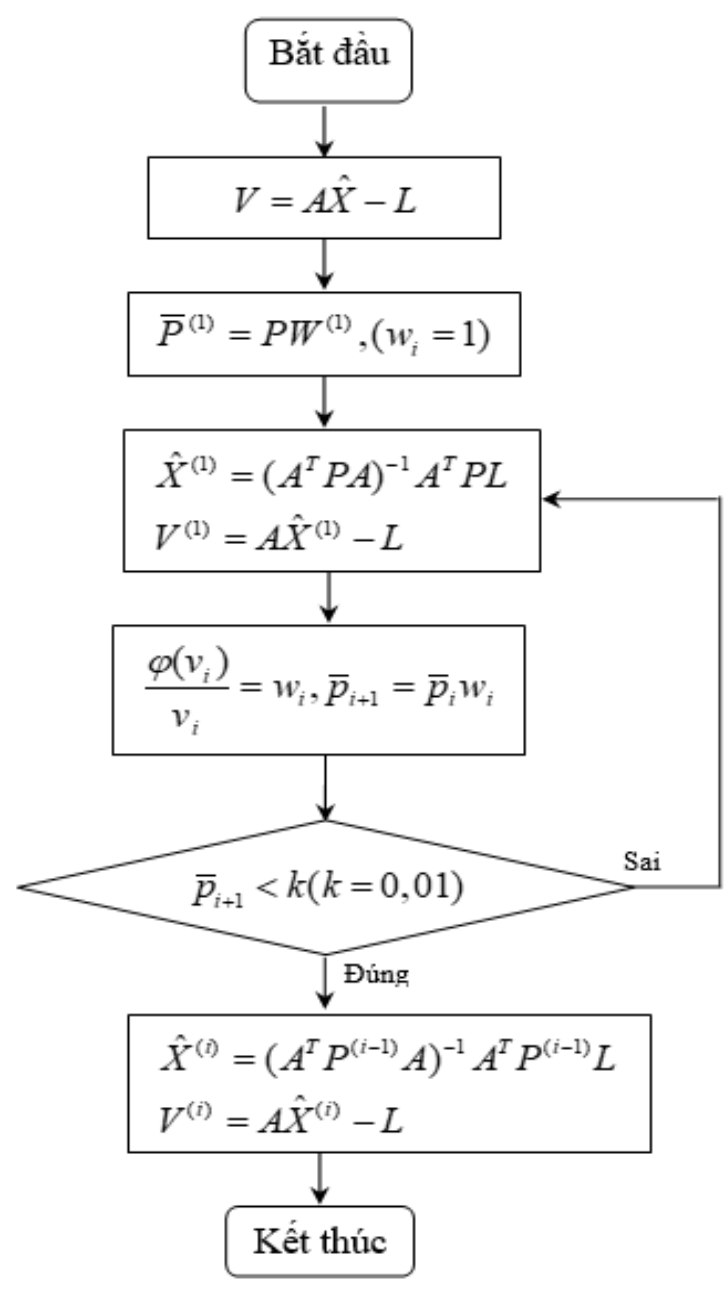

Hình 2. Quy trình thay thế chọn trọng số theo phưong sai hậu nghiệm.

Phân tích số liệu ở các Bảng 1, 2 nhận thấy:

- Số hiệu chỉnh của các trị đo không có sai số thô ít nhiều đều bị ảnh hưởng bởi trị đo có sai số thô.

- Các trị đo có chứa sai số thô có trọng số tiến về “ 0 ”, đây cũng chính là nguyên lý cơ bản của ước lượng vững.

- Số hiệu chỉnh sau bình sai kết hợp phát hiện sai số thô bằng ước lượng vững tương đối gần với sai số đưa vào khảo sát. Trị đo càng được đo chính xác thì khi có sai số thô càng xác định được lượng sai chính xác hơn. Ngoài ra, giá trị của sai số thô còn bị ảnh hưởng bởi mô hình bình sai.

- Trong thực nghiệm này, điều kiện hội tụ là khi trọng số của trị đo nhỏ hơn 0,01 . Tuy nhiên, nếu dữ số liệu ban đầu không chính xác (không tuân theo phân bố chuẩn Gauss) thì phương pháp tìm sai số thô này sẽ bị phân tán, tức không hội tụ.

\section{Kết luận}

- Phương pháp xác định sai số thô bằng ước lượng vững theo phương sai hậu nghiệm có lý thuyết rõ ràng nên dễ thực hiện. Đặc biệt có hiệu quả đối với mạng lưới lớn có nhiều trị đo thừa.

- Phương pháp này xác định được vị trí và gần đúng giá trị của sai số thô mà không mất nhiều thời gian, chỉ qua một lần bình sai là có kết quả nên rất thuận lợi khi lập chương trình tính toán.

- Giá trị sai số thô không được xác định chính xác là do sai số của phép đo, sự tương quan của các trị đo sai số mô hình bình sai gây ra.

- Hoàn toàn có thể sử dụng phương pháp này trong thực tế, phục vụ sản xuất.

\section{Đóng góp của các tác giả}

Phạm Quốc Khánh - lên ý tưởng, viết bản thảo bài báo; Nguyễn Thị Kim Thanh - thu thập dữ liệu, đánh giá và chỉnh sửa.

\section{Tài liệu tham khảo}

Baselga, S., (2007). Global optimization solution of robust estimation. Journal of Surveying Engineering-ASCE, 133(3).

Guo, J., Ou, J., Wang, H. (2010). Robust estimation for correlated observations: Two local sensitivity-based downweighting strategies. $J$. Geod., 84, 243-250.

Li Deren, Yuan Xiuli. (2012). Error Processing and Reliability Theory. Wuhan University Press, tiếng Trung Quốc.

Luu Anh Tuan, Le Ngoc Giang. (2016). Application of robust estimation in geodetic network. International symposium on geo-spatial and mobile mapping technologies and summer school for mobile mapping technology, 213218.

Quoc Khanh Pham. (2016). Research into method used for detecting geodetic non-random errors based on adjustment canculus. International symposium on geo-spatial and mobile mapping technologies and summer school for mobile mapping technology, 55-59.

Wang Xinzhou, Tao Benzao,Qiu Weining, Yao Yibin. (2006). Advanced surveying adjustment. Surveying and Mapping Publishing House. tiếng Trung Quốc. 
Wang, Y, Zhu, X. X. (2016). Robust Estimators for Multipass SAR Interferometry. IEEE Trans. Geosci. Remote. Sens., 54, 968-980.

Yang Yuanxi, Xu Tianhe, Song Lijie. (2005). Robust estimation of variance components with application in global positioning system network adjustment. Journal of Surveying Engineering, 131(4).
Yetkin, M. (2018). Application of robust estimation in geodesy using the harmony search algorithm. J. Spat. Sci., 63, 63-73.

Yetkin, M., Berber, M. (2014). Implementation of robust estimation in GPS networks using the Artificial Bee Colony algorithm. Earth Sci. Inform., 7, 39-46. 